\title{
Image Processing and a Virtual Restoration Hypothesis for Mosaics and Their Cartoons
}

\author{
Mariapaola Monti ${ }^{1}$ and Giuseppe Maino ${ }^{1,2}$ \\ ${ }^{1}$ Faculty of Preservation of the Cultural Heritage, University of Bologna, \\ Ravenna site 5, via Mariani, Ravenna, Italy \\ mariapaola.monti@studio.unibo.it, giuseppe.maino@unibo.it, \\ ${ }^{2}$ ENEA, Italian National Agency for New Technologies, Energy and Sustainable Economic \\ Development, 4, via Martiri di Montesole, Bologna, Italy \\ giuseppe.maino@enea.it
}

\begin{abstract}
We present some results of image processing relevant to mosaics. In particular, the three-dimensional image of a mosaic is obtained by means of a laser scanning technique, then adapted to a GIS software, describing main characteristics of single tesserae. Furthermore, the virtual restoration of Ravenna mosaic cartons is performed and shown by a few examples.
\end{abstract}

Keywords: Mosaics, laser scanning, Geographical Information System, virtual restoration, cartoon.

\section{Introduction}

Cesare Brandi in his Theory of Restoration (1963) [1] stated that "restoration is the methodological moment of recognizing a work of art in its physical consistency and in its dual aesthetical and historical polarity, in view of its transmission in the future", that "it must aim at restoring the potential unity of the work of art, provided this is possible without making an artistic or historical fake, and without erasing any trace of the passing of the artwork through time", that "we can restore only the material of the work of art" and that "the restoration, in order to be a legitimate operation, should not assume neither the time to be reversible nor the abolition of history".

Cesare Brandi developed his theory of restoration over the years (1939-59), when he directed the Istituto Centrale del Restauro (ICR), founded in Rome in 1939 by himself, designed by Giulio Carlo Argan, in order to set the task of restoration on a scientific basis and on a multidisciplinary approach, which involves the collaboration of art historians, restorers, and laboratory technicians.

These principles, as intended by the author, clearly referred not to the virtual restoration but to the real restoration, which concerns the artwork substance. Today they are considered as fundamental principles in any traditional restoration intervention. These principles were developed between 1939 and 1959, when virtual restoration did not exist, as we can begin to talk about digital era only since the 60s of XX century [2]. 
However, they - summarized as respect for the aesthetic and historical aspects, compatibility of materials, recognizability of the intervention, reversibility of the materials and minimal intervention - must also be applied to virtual restoration (which must be a legitimate intervention and not a work of fiction or a display of skill), although this consists only in a restoration of an aesthetic nature, which does not involve the material of the work of art. For this reason, the principle of compatibility of materials is not applicable, but all the others remain valid.

Therefore the restoration techniques used via computer, initially called "Electronic Restoration", now commonly called "Virtual Restoration", would be more correctly called "Digital Iconological Restoration" because it is a digital processing of the computer image (in Greek cíkóv) of a work of art [3]. Generally this digital processing is designed to improve the visual and aesthetic aspects of an artwork or to a hypothetical reconstruction of it, which allows for greater readability.

In this sense, the virtual restoration does not compete with the real one but it supports and assists, as it envisages the possible outcomes (such as when you must choose between different methods of intervention) and observes the same rules (arbitrary rebuilding or reconstruction are not permitted). It also allows you to obtain a usable image of the work of art, when the same is not materially restorable because of high costs or a condition of excessive fragility of the materials.

Simplifying, we can say that - while the traditional restoration aims primarily at extending the life of the product -, the virtual restoration aims at achieving better readability of the information contained in the artwork.

We must never forget that a restoration intervention, as in itself always traumatic for the piece of art, should be carried out only in case it is necessary for the survival of the artwork; therefore, when it is necessary to improve the readability of the image, then traditional restoration can be replaced by virtual restoration, thereby conserving the integrity of the original materials. In other words, you can place side by side the fragmented piece and its reconstructed image, cleaned up or rebuilt, but only where it can be done without falling into arbitrary solutions.

Today, "virtual restoration" also means digital improvement, thanks to special algorithms and mathematical relationships, of the images obtained by diagnostic investigations on works of art and antique documents (photographic analysis, X-ray, UV, IR tests), in order to facilitate their comprehension [4]. The advantages of a virtual restoration rely on its specificity compared to the manual method: A digital image, "clone" of the real one, may be altered, duplicated, restored many times without jeopardizing or damaging the real work of art. So, you can work with the maximum freedom of action, in some cases even putting aside the principles of traditional restoration.

Intervention attempts may be different and may also be modified subsequently: Each phase of the intervention may be registered at a different level in a photo editing program like Adobe Photoshop, Corel PhotoPaint or GIMP. In a certain sense this allows for the reversibility of the intervention and immediate comparisons between the different phases and possibly among several operational choices.

The reconstruction hypothesis digitally carried out may also be mimetic, maybe carried out on a different level compared to the original image in order to guarantee their identification. It is important, though, that they be justified by a philological analysis of the piece. 


\section{Three-Dimensional Laser Scanning of Mosaics}

Mosaics have an intrinsic three-dimensional structure due to finite size, shape, position and orientation of tesserae in order to produce particular effects by light reflection, formation of shadows, etc, as well as an extrinsic one since they are often located on curve surfaces such as vaults, domes, pillars and so on. Unfortunately, the usual photographic documentation does not account for these peculiar characteristics and propose a necessarily planar image of mosaics, thus neglecting important information that, in the case of surveys preliminary to restorations, for instance, is rendered in graphics by means of conventional notations.

Therefore, use of 3D laser scanner has been proposed to overcome these difficulties and provides archaeologists, art historians and museums keepers with suitable tools for a better knowledge and representation of mosaics. The instrumentation described in the previous sections has been utilized on samples and large mosaics such as those in the Basilica of St.Apollinare Nuovo in Ravenna (Italy).

A main difficulty arises from lack of data in some regions in correspondence with tesserae borders and dark colour elements - in particular for black tesserae where the laser light is completely absorbed and dark green ones - resulting from the dominant glass material in mosaics composition since its lucid and compact surface reflects the light in such a way that it is only partly detected by the optical sensors of the instrument. This disturbing phenomenon is often generated in scanning bronze works, which - to overcome this limitation - were, in some cases, treated with powder or spray opacifiers provided their removability. It was considered necessary, in our case, sprinkle the mosaic surface of powder, thus making possible the acquisition of a sufficient amount of points from all the tiles.

In the attempt to compensate for any gap in the data cloud configured in the first scan tests, it was thought to capture the same portion of the surface several times, by inclining the laser emitter at different degrees. For these operations, the manufactured support which allows a shift $(50 \mathrm{~cm} . \times 35 \mathrm{~cm}$.) was fixed to a rotatable mechanical base, connected to the computer and operated directly by the software processing of the clouds of points, in order to define precisely the angle of inclination of the surface to be collected with respect to the scanner.

After completing the acquisition of the mosaic, taken to an inclination of $0^{\circ}, 15^{\circ}$, $30^{\circ}, 45^{\circ}$ and $70^{\circ}$, respectively, it was made the realignment of the five resulting clouds of points. By means of the Polygon Editing Software Tool , supplied as a standard accessory to the scanner by Minolta, one has identified counterparts of the points in each cloud, to be aligned through suitable roto-translations. A subsequent merging feature has produced a single mesh of points, where the lack of information derived from the cloud at $0^{\circ}$ scan is partially compensated by the superimposition of the other clouds. The final result is shown in Figure 1.

As for the scheduling of a real campaign of three-dimensional relief for mosaics of large dimensions, a few issues that could be solved thanks to the rapid evolution of these technologies have to be carefully planned as previously shown, resulting laser scanning a useful tool for documentation of mosaics. Moreover, reverse engineering methods are interesting for several reasons. The creation of virtual models of small portions, representing compositions where they belong, would permit to carry out 


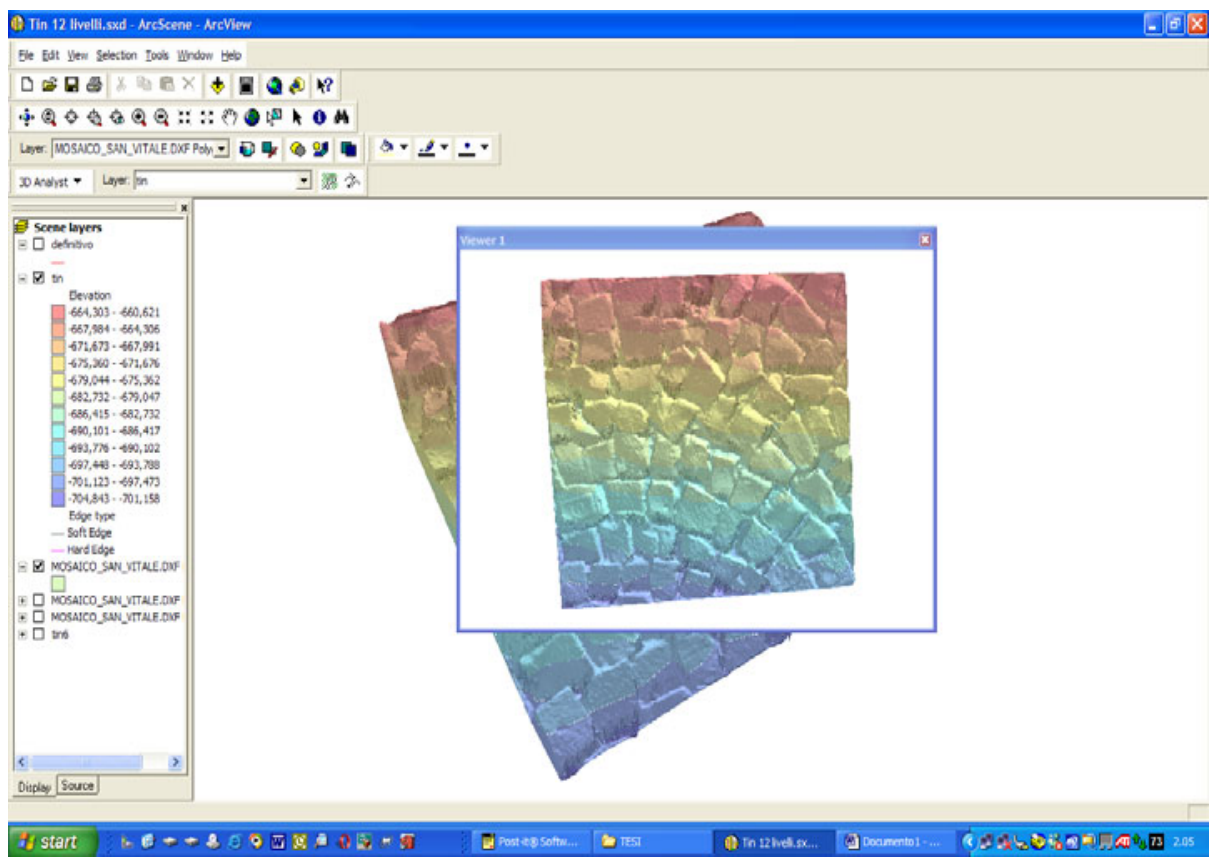

Fig. 1. Three-dimensional rendering of a mosaic sample

comparative investigations of mosaic cycles, leading to quantify similarities and differences in terms of processing and surface rendering of the mosaics themselves. From the virtual model one can perform accurate measurements on the size of the tesserae, their distance, position and orientation, even their projection with respect to the support.

Figure 2 represents the screen view of a geographical information system (GIS) namely, ArcGis - where the three-dimensional relief of the mosaic has been implemented. Therefore, one can exploit all the features of a GIS software to perform numerical analyses of the whole surface, the position, shape and orientation of single tiles, average values and so on, and create a valuable geo-referenced database documenting in great details the investigated mosaics. As an example, Figure 2 shows a precise measurement of the orientation of a single tessera with respect to the plane of the support.

The application of GIS in a mosaic has been then tested by the integration of data from three-dimensional laser scanning, using software such as Rhinoceros 3.0 and ArcGis 8.01. The first program is a model of land and is used mainly to handle the file format. Stl directly derived from scanning laser data. It has been necessary to convert this format in order to be able to import data into the GIS system, which is not configured to manage the files of this type. The file format .Stl was then converted into a file .Dxf (drawning exchange file), a standard for CAD vector systems. By this way, it was possible to import the model of the mosaic inside ArcGis. The file can then be configured as a set of lines and polygons that describe the geometry of the object and its morphological characteristics. 


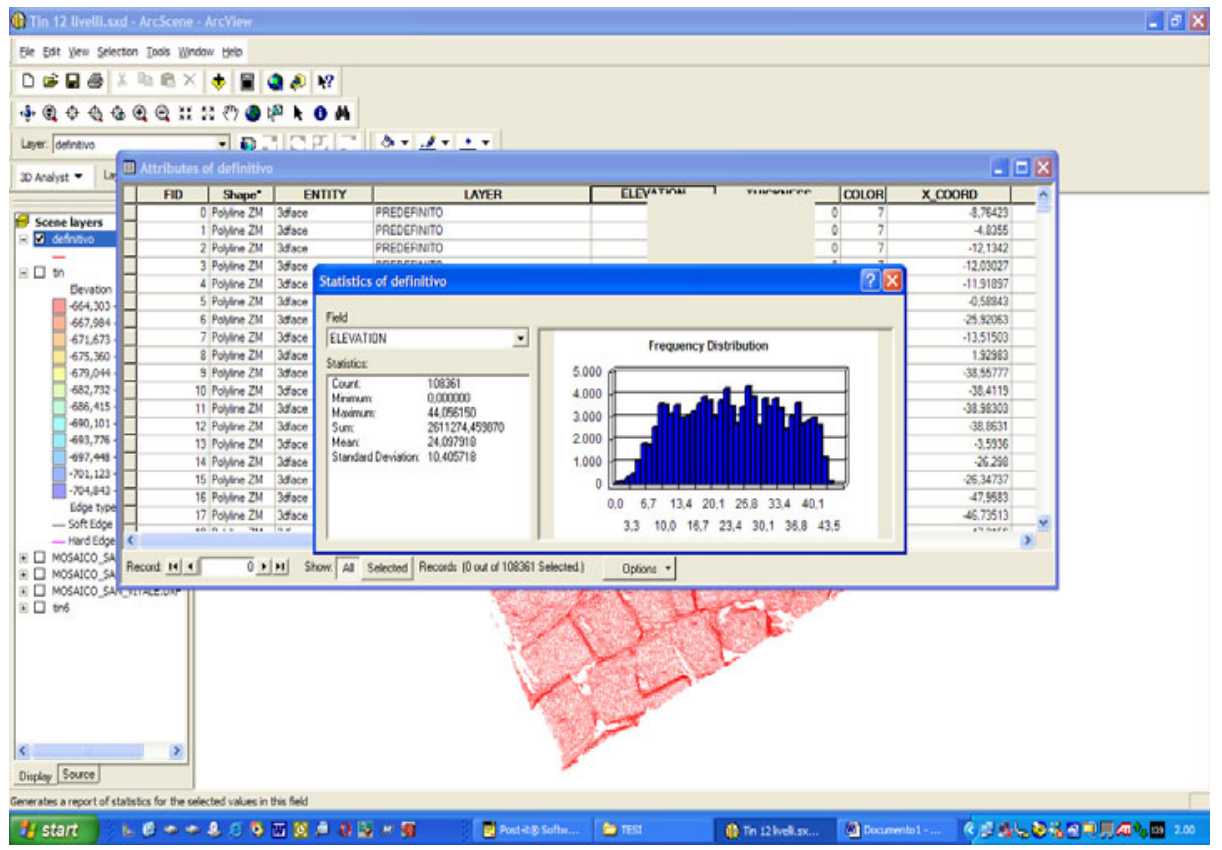

Fig. 2. Screen view of a GIS for mosaics

With these data it has been feasible to build a TIN based on the elevations of geometric primitives. Represented graphically by means of chromatic intervals, the file provides clear and immediate information in the performance of the quantitative measurements of 'tessellato'. The GIS also includes the possibility of statistical analysis on which one can draw in real time characteristics such as maximum and minimum value of the selected fields (height in this case) and the standard deviation, so these precise calculations and immediate morphological study on the surface are easier and quicker than in traditional mappings, thus allowing archaeologist and art historian to have objective data on which to base their thoughts and assumptions on the interpretation of mosaics.

\section{Virtual Restoration of Mosaics Cartoons}

In the particular case of cartoons representing mosaics, virtual restoration is carried out not in order to prefigure a real restoration, but to enhance and extract information contained in these artworks regarding the grid and the colors of the mosaic with the aim of its reproduction. Therefore, this virtual restoration will not follow the classical criteria of restoration, which would impose the ban to reconstruct the missing parts, although in this case an arbitrary reconstruction is not involved, since it can refer to the original mosaics from where the cartoons were taken from.

Mosaic cartoons are tempera paintings which represent ancient mosaics traced tile by tile. The aim is to create printable replacements of the original cartoons, which 
may be used in daily teaching activities and which contain as much as possible clear and complete information. In this way the most important cartoons could be taken away and stored in appropriate ways without depriving students from them anyway.

Since specific programs for virtual restoration were not developed, commercial softwares are generally used for vector or bitmaps graphics, in this case, Adobe Photoshop CS. Prior to the real intervention, an adequate CMS system (Color Management System) should be used. Thanks to the use of a colorimeter or a spectrophotometer, CMS can coordinate the gamut, that is the color spaces of different output devices (such as printer and monitor), in order to have the colours of the scanned images correspondent as much as possible to one another and to those of the original artwork.

The process of virtual restoration on the mosaic cartoons consists of the following phases:

$\checkmark$ digital data acquisition,

mosaicking,

- elaboration (balance, cleaning, reconstruction, extraction of the grid),

- electronic filing.

Of course, memory of each step and phase of digital processing must be kept in a different level of a Photoshop or GIMP file (with a "psd" extension), able to store several overlying images, saving them without compression and therefore without losing quality.

The digital acquisition is generally performed using a digital camera, the resolution of which today usually ranges from 8 to 14 Megapixels (millions of pixels). To obtain a higher resolution we can also use scanners or digital backs, namely a camera in which the traditional photosensitive film is replaced by a CCD (charge-coupled device) or CMOS (complementary metal-oxide semiconductor) able to capture the image, transforming it into an electrical signal.

If the cartoon to be photographed is very large it can be acquired through several shots, overlapping the edges which then must be reassembled through mosaicking, in order to obtain a resolution of at least 500 or 600 ppi (pixels per inch) on the real size, required to display a screen magnification of all details and to make extremely high quality prints. The mosaicking consists in the perspective rectification of several shots that are then reassembled to form the image of the entire object, perfectly matching the margins.

Once reassembled, not only do the high-resolution photographs allow you to gain a better understanding of the artwork, but they also help to assess the conservation status and identify any previous restoration works.

A first elaboration of the image obtained in this way is to balance brightness and contrast, and to regulate dominant colors, using the "Curves" tool in Photoshop in order to make the picture as similar as possible to the original one, since each acquisition inevitably causes overexposure or underexposure and color toning, due to lighting conditions and the characteristics of the sensors used.

In order to adequately perform these corrections, photos must be carried out by placing a color scale next to the cartoon. By doing so, we can have a reliable reference so as to assess the deviation of color and brightness values of the picture from those of the original one. 
For instance in figure 3 we see that the scanned image appears too pale, blurry and slightly turned towards a red color. This is the photograph of the painting by Alessandro Azzaroni representing the pair of doves from the Mausoleum of Galla Placidia. Instead in figure 4 (left) the same image was balanced on the basis of the colors shown by the original mosaic.

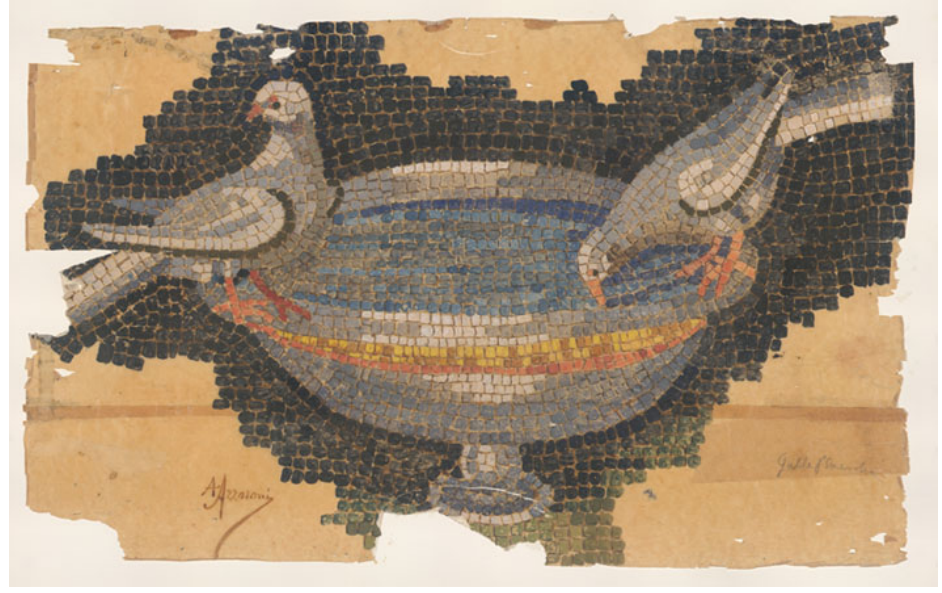

Fig. 3. The painted cartoon by Alessandro Azzaroni representing the pair of doves from the Mausoleum of Galla Placidia

In this way it was possible not only to correct the imperfections due to image acquisition, but also the changes of color of the tesserae, due to alterations of paper color (photochromic degradation) over time; indeed, the new yellow-orange dominant color of paper can be seen through the thin layer of the tempera.

Then, if necessary, you can perform cleanup operations (removal of stains) and intensification of the faded lines. This is a recovery of information which is not always possible to carry out with a traditional restoration intervention. Thanks to the use of IR and UV photography, it is also possible to separate and give back distinctness to any palimpsests.

The reconstruction of missing parts, as we see in figure 4, consists in the use of the image's whole parts in order to recreate the shape of the lost areas, only where it is possible to do so with certainty, therefore for very small gaps and also for much larger ones if there are other copies of the artwork to be restored: in our case we keep the original mosaics on which the cartoons are based.

In addition to the overlapping of different levels corresponding to different phases of work, also the areas of the cartoon where digital reconstruction has been carried out are highlighted by a red edge. The gold tesserae at the base of the cup took on a greenish color in the cartoon, probably due to an alteration of porporina, Pigment consisting of a mixture of metal powders, usually a golden color, easily alterable in contact with air humidity, especially in tempera paintings; in order to give them a color closer to the original we used the "Color replacement" tool.

Conversely, the golden band that decorates the cup was made with yellow, orange and ocher tempera, therefore it did not suffer any alteration. 

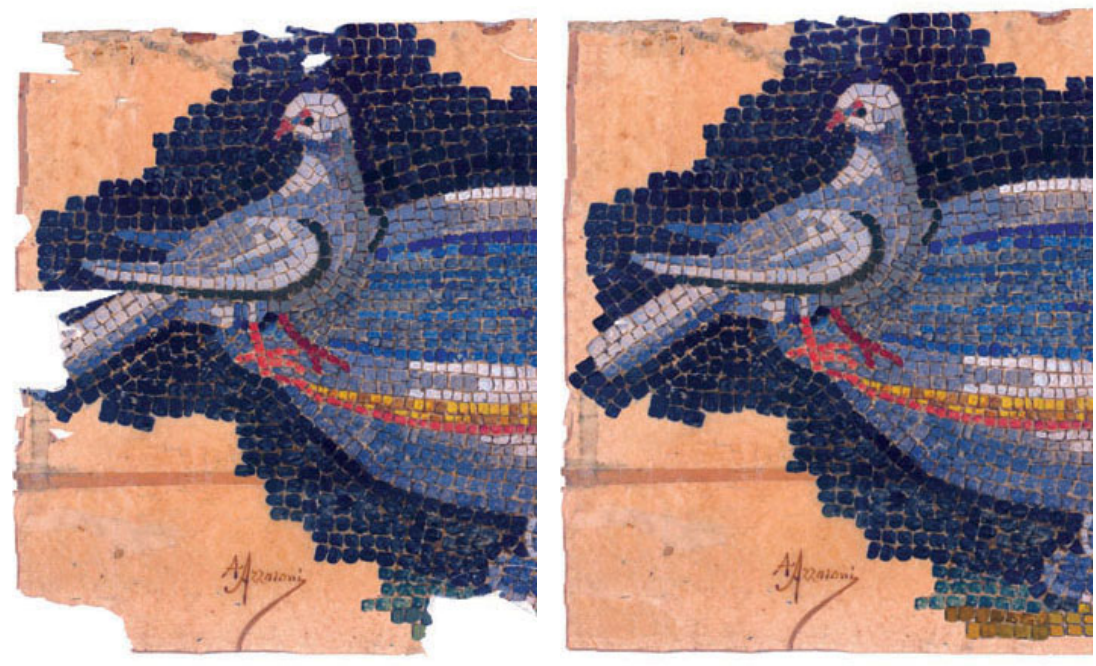

Fig. 4. Drinking doves from Mausoleum of Galla Placidia - cartoon by Alessandro Azzaroni (detail)

The extraction of the grid shown in figure 5, which can be useful to make copies in mosaic, was carried out by using the "Find edges" tool on the image of the restored cartoon, transformed into "Grayscale"; then the contrast was enhanced by using "Curves" to place greater emphasis on the edges of the tesserae, which were then manually cleaned one by one with the "Brush" tool, as the color variations inside were also highlighted, albeit mildly, using the "Find edges" tool.

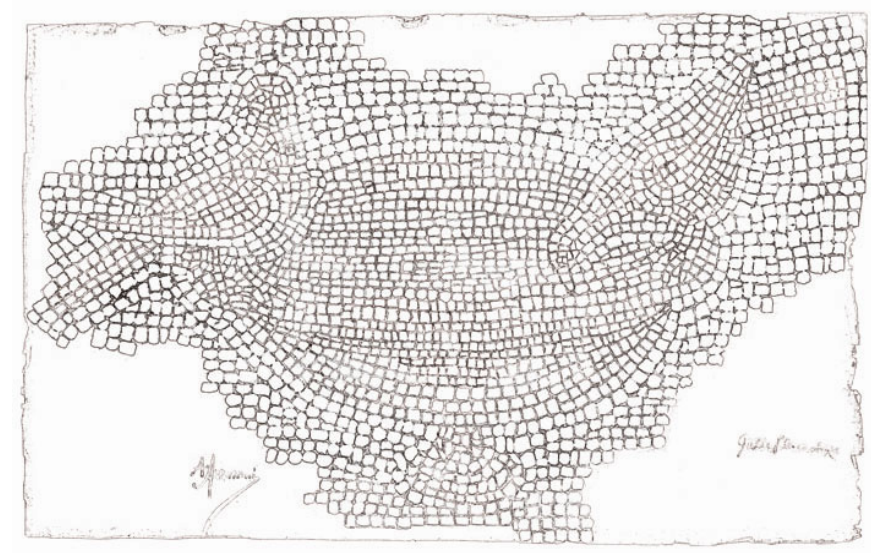

Fig. 5. Extraction of the grid from the mosaic cartoon of figure 4

The last phase of intervention consists in electronic storage of high and low resolution images, in order to keep the acquired images and all subsequent elaborations, foreseeing both their consultation and any new virtual restoration based 
on different principles. Saved high-resolution images may be used to make high quality prints for periodicals or magazines and for sales. Low resolution ones instead may be used to make on-line catalogues or user-friendly virtual museums. All this should be carried out in order to promote the knowledge of Ravenna's so characteristic heritage formed by mosaics and their cartoons.

During this virtual restoration project, four cartoons representing Ravenna's mosaics belonging to the Severini Institute were photographed.

The images were acquired with a Nikon D70 digital SLR camera, Nikon DX CCD sensor with 6.1 megapixel $(23.7 \times 15.6 \mathrm{~mm})$ and a Nikkor AF-S lens (AutofocusSilent, which uses an SWM technology, Sonic Wave Motor, to make it quieter and faster focusing), and in some cases with the addition of a Hoya 80A filter (light blue), used to correct the prevailing warm tones that often feature pictures taken indoors in artificial light.

Anyhow, any warm and cold tones produced by the filter were subsequently digitally corrected thanks to the color and grey scale used in every shot. The lighting was obtained with the use of flash combined with natural daylight from the window of one of the classrooms in the Severini Institute. The color space used by the camera is sRGB (standard RGB created jointly by HP and Microsoft in 1996) and the size of the photographs is $3008 \times 2000$ pixels $(25.47 \times 16.93 \mathrm{~cm}$ at $300 \mathrm{ppi})$.
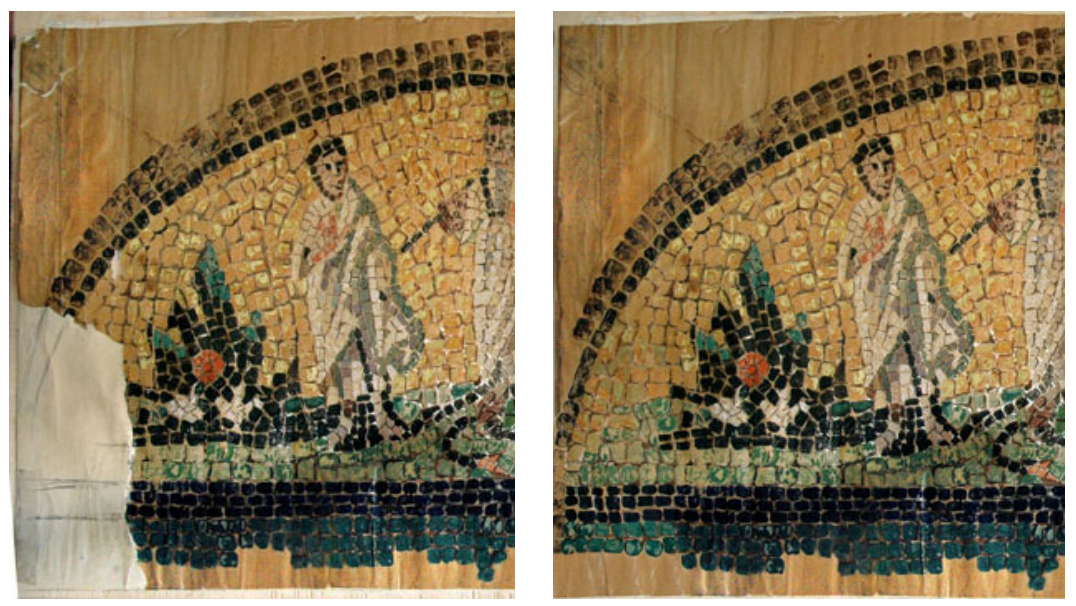

Fig. 6. Lunette with Christ and Apostles from S. Apollinare Nuovo - cartoon by Zelo Molducci (detail).

Given the size $(104 \times 70 \mathrm{~cm})$, cartoon $\mathrm{N}^{\circ} 54$ was photographed in two shots, partially overlapping, that, after color, brightness and contrast balance, were subjected to perspective rectification and mosaicking. The resulting image has a resolution of 90 ppi at actual size. Then the mosaic grid was extracted from the reconstructed image.

So, for photographs of cartoon $\mathrm{N}^{\circ} 54$ we had to carry out a recomposition process by mosaicking, but not a digital reconstruction intervention of the missing parts, since this picture is preserved intact; on the contrary, cartoons $\mathrm{N}^{\circ} 84,88$ and 91 , which are smaller, were acquired with only one shot, but since they had different blanks, they were subjected to a mimetic reconstruction of the lost fragments, as shown in figs. 4 and 6 . 
For image of the cartoon $\mathrm{N}^{\circ} 84$ we were able to obtain a resolution of 90 ppi at actual size $(50 \times 40 \mathrm{~cm})$, while for those of cartoons $\mathrm{N}^{\circ} 88$ and 91 we have achieved a resolution of $100 \mathrm{ppi}$ at actual size (respectively $68 \times 38.5 \mathrm{~cm}$ and $60.8 \times 46.6 \mathrm{~cm}$ ).

Interventions like these, possibly with the use of tools that improve resolution, carried out on all cartoons with greater historic value, would allow you to preserve the originals from daily use, without subtracting the information contained inside them, but rather improving and spreading them via prints or accessible on-line data banks.

\section{References}

[1] Brandi, C.: Teoria del restauro, Edizioni di Storia e Letteratura, 1st edn., Roma (1963); consulted edition: Piccola Biblioteca Einaudi, Torino, pp. 6-8, 26 (1977)

[2] Ferrarini, E., Staltari, E.: Scrittura ed immagini: un'ipotesi di restauro virtuale. Le Médiéviste et l'Ordinateur. Histoire médiévale, informatique et nouvelles technologies (41) (2002)

[3] Bennardi, D., Furferi, R.: Il restauro virtuale. Tra ideologia e metodologia, Edifir, Firenze, p. 13 (2007)

[4] Gonzales, C., Woods, E.: Digital Image Processing. Addison-Wesley, N.Y (1993) 